\title{
Nasal dissemination of a single-clone IgH-rearranged conjunctival MALT lymphoma through the nasolacrimal duct: A case report
}

\author{
CHUNG-YU HSIEH ${ }^{1}$, YI-PING LIAO ${ }^{1}$, CHIA-CHE WU ${ }^{1}$, SHENG-PO YUAN ${ }^{1}$, JENNIFER HUI-CHUN HO ${ }^{2,3,4}$, \\ RACHEL ROAN ${ }^{5}$, PHUI-LY LIEW ${ }^{6,7}$, MING-TANG LAI ${ }^{1}$ and FEIPENG LEE ${ }^{1}$ \\ Departments of ${ }^{1}$ Otolaryngology and ${ }^{2}$ Ophthalmology, Wan Fang Medical Center, Taipei Medical University, Taipei 11698; \\ ${ }^{3}$ Graduate Institute of Clinical Medicine, Taipei Medical University, Taipei 11031, Taiwan R.O.C.; \\ ${ }^{4}$ Institute of Engineering in Medicine, University of California, La Jolla, San Diego, CA 92093; ${ }^{5}$ Post-Baccalaureate Health \\ Professions Program, UC Berkeley Extension, Berkeley, CA 94704, USA; ${ }^{6}$ Department of Pathology, Shuang Ho Hospital, \\ Taipei Medical University, New Taipei City 23561; ${ }^{7}$ Department of Pathology, School of Medicine, \\ College of Medicine, Taipei Medical University, Taipei 11031, Taiwan R.O.C.
}

Received March 26, 2015; Accepted May 5, 2016

DOI: $10.3892 / \mathrm{ol} .2016 .4700$

\begin{abstract}
The aim of the present study was to report a rare case of single-clone, immunoglobulin heavy chain (IgH)-rearranged mucosa-associated lymphoid tissue (MALT) lymphoma in the conjunctiva, with nasal cavity dissemination through the nasolacrimal duct. A 24-year-old female was diagnosed with MALT lymphoma of the nasal cavity at the Department of Otolaryngology, Wan Fang Medical Center, Taipei Medical University (Tapei, Taiwan) in October 2008. A biopsy of the relapsing conjunctival lesion revealed a MALT lymphoma by pathological staining, while a single-clone, IgH-rearranged tumor lesion in the nasal cavity and conjunctiva was confirmed using continuous sinus computed tomography scans and polymerase chain reaction. Tumor lesions were negative for Helicobacter pylori and Chlamydia infection, but exhibited bilateral neck lymph node dissemination. A combination of radiation therapy (a total dosage of 46.8 Gray, in two phases covering the left lacrimal sac, nasal cavity and bilateral neck region) and topical ciprofloxacin plus steroid (0.3\% ciprofloxacin 4 times a day and betamethasone eye ointment before sleep for 1 month) was provided as an effective therapeutic strategy, and no recurrence was found in the next 3 years. The nasolacrimal duct serves as a channel for conjunctival tumor spreading and is easily neglected. IgH-involved translocation in MALT
\end{abstract}

Correspondence to: Dr Ming-Tang Lai, Department of Otolaryngology, Wan Fang Medical Center, Taipei Medical University, 111 Hsing-Long Road, Section 3, Taipei 11698, Taiwan R.O.C.

E-mail: wfentlmt@gmail.com

Key words: immunoglobulin heavy chain translocation, mucosa-associated lymphoid tissue lymphoma, nasal cavity, nasolacrimal duct, conjunctiva lymphoma is a factor in the progression of the disease, and aggressive combination therapy is essential for a high-risk, disseminated IgH-rearranged MALT lymphoma.

\section{Introduction}

Ophthalmic lymphomas account for $5-10 \%$ of extranodal lymphomas, and extranodal marginal zone B-cell lymphomas of mucosa-associated lymphoid tissue (MALT) are the leading subtype among ophthalmic lymphomas $(1,2)$. However, only a few cases of sinonasal MALT lymphomas were reported in the past 20 years $(3,4)$. The present study reports a rare case of a MALT lymphoma in the conjunctiva, lacrimal sac and nasal cavity, which occurred 4 years following the spontaneous regression of conjunctival atypical lymphoid hyperplasia. The pathological features, imaging study and a gene arrangement test were used to diagnose a single-clone MALT lymphoma in the conjunctiva and nasal cavity. The role of the immunoglobulin heavy chain $(\operatorname{IgH})$ gene rearrangement and atypical infection in MALT lymphoma was also discussed. A successful combination treatment, with local radiotherapy and topical antibiotics plus steroid for a disseminated MALT lymphoma, was also demonstrated in the present study.

\section{Case report}

A 24-year-old female initially presented to the Department of Ophthalmology, Wan Fang Medical Center, Taipei Medical University (Taipei, Taiwan) in November 2004 with a painful salmon pink-like lesion in the left inferior conjunctiva that had lasted for several months (Fig. 1A). A tissue biopsy was performed by an ophthalmologist and sent to the Department of Pathology, Wan Fang Medical Center, Taipei Medical University, where it was formalin-fixed and paraffin-embedded. Immunohistochemical staining was performed using an automated stainer (BenchMark XT, Ventana Medical Systems, Inc., Tucson, AZ, USA) with 4- $\mu \mathrm{m}$ thick sections from formalin-fixed and paraffin-embedded 
A

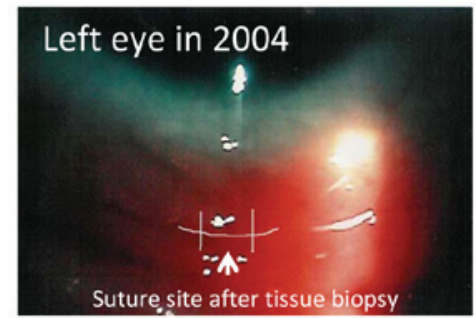

D
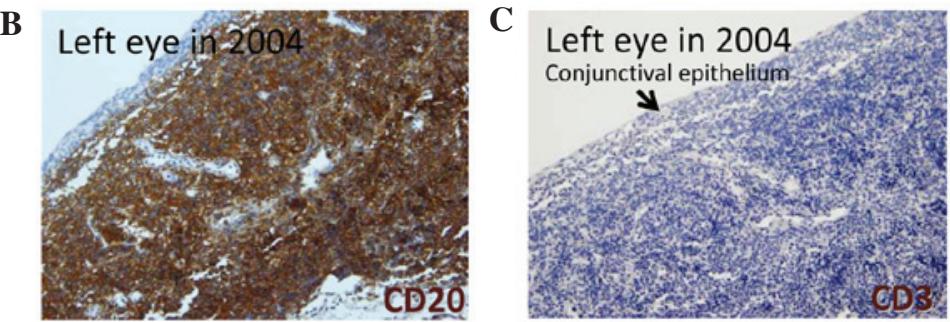

$\mathbf{E}$
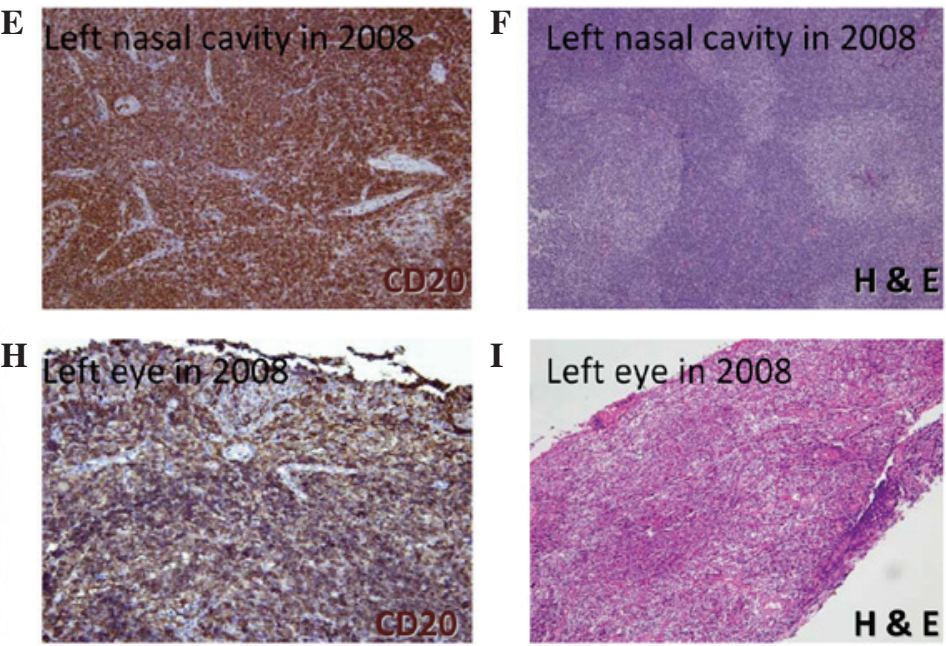

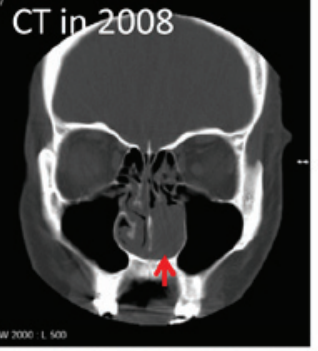

G

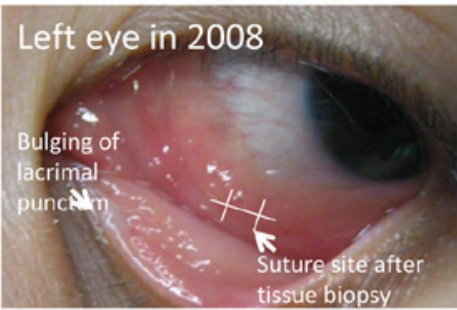

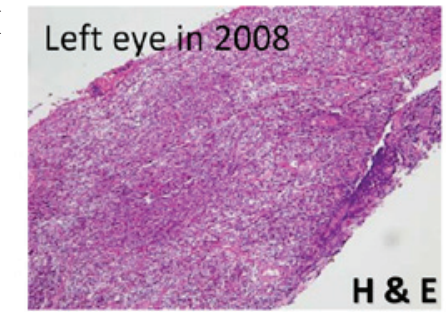

Figure 1. (A) In November 2004, the initial presentation was a salmon pink-like patch lesion at the left lower conjunctiva. Immunohistochemical staining revealed that the lesion was (B) positive for CD20 and (C) negative for CD3 staining. (D) In October 2008, a soft-tissue mass in the left nasal cavity with bone destruction was detected by a sinus computed tomography scan. Histopathological staining revealed the diffuse proliferation of (E) CD20-expressing atypical lymphoid cells and (F) surrounding lymphoid follicles. (G) Recurrent salmon pink-like lesion of the left inferior conjunctiva with bulging of the lacrimal punctum was also found in 2008. A conjunctival biopsy demonstrated the (H) atypical B-cell infiltration and (I) lymphoma involvement with epitheliotropism. CD, cluster of differentiation.

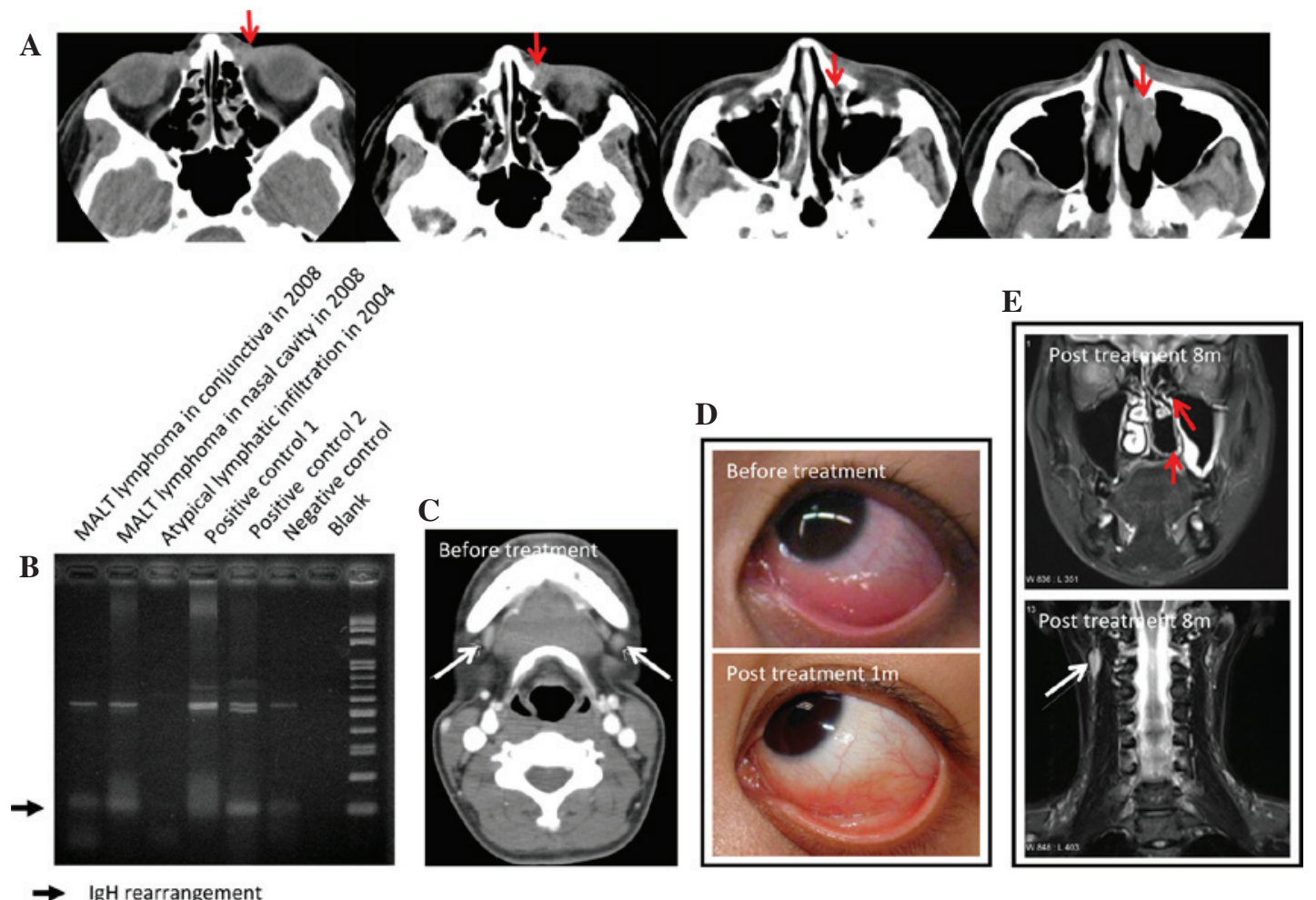

Figure 2. (A) Continuous imaging sections from the inferior orbit to the nasal cavity on sinus CT scan showed the soft-tissue mass extension in lacrimal duct and sac. (B) Gene rearrangement of immunoglobulin heavy chain by polymerase chain reaction illustrated a single-clone disease of mucosa-associated lymphoid tissue lymphoma in the nasal cavity and conjunctiva. (C) Lymph node enlargements in the bilateral submanibular spaces and neck region were noted on the CT scan. (D) Conjunctival mass was regressed after administering topical ciloxan eye drops and betamethasone eye ointment for 1 month. (E) Tumor mass in nasal cavity and lacrimal sac were invisible (upper) and one stationary neck lymph node was noted following radiotherapy (bottom). CT, computed tomography. 
tissues. Tissue sections were deparaffinised and rehydrated in graded alcohols and xylene. After retrieval by the autoclave retrieval technique (10 $\mathrm{mM}$ citric acid buffer; $10-20 \mathrm{~min})$ and inhibition by endogenous peroxidase activity $\left(0.3 \% \mathrm{H}_{2} \mathrm{O}_{2}\right.$; $5 \mathrm{~min}$ ), sections were incubated with primary antibodies. The primary antibodies used were CD20 monoclonal mouse (dilution, 1:200; catalog no., CM004; Biocare Medical, Concord, CA, USA) and CD3 monoclonal mouse (dilution, 1:200; catalog no., NCL-L-CD3-565; Leica Microsystems GmbH, Wetzlar, Germany). Negative controls were performed by omitting primary antibodies. After washing with Tris buffer, sections were incubated for $30 \mathrm{~min}$ with a polyclonal secondary antibody against mouse and rabbit from the ultraView Universal DAB Detection Kit (dilution, 1:100; catalog no., 760-500; Ventana Medical Systems, Inc.), re-incubated with $100 \mu \mathrm{g} / \mathrm{ml}$ peroxidase-conjugated streptavidin, and colonized with $0.02 \%$ 3,3'-diaminobenzidine tetrahydrochloride (0.05M Tris-HCL buffer with $0.03 \% \mathrm{H}_{2} \mathrm{O}_{2}$ ). The sections were counterstained with hematoxylin, dehydrated and mounted. The surgical specimens revealed atypical B-lymphoid hyperplasia (Fig. 1B and C). Spontaneous tumor regression was observed in the following 6 months.

The patient visited the Department of Otolaryngology, Wan Fang Medical Center, Taipei Medical University in October 2008 complaining of left nasal pain that had lasted for 2 months. A lobulated left nasal tumor (18.8x24.6x37.4 mm in size) with a bony destruction was noted on a sinus computed tomography (CT) scan (Fig. 1D). Two incisional biopsies performed by an otolaryngologist and sent to the Department of Pathology, Wan Fang Medical Center, Taipei Medical University confirmed a stage II mucosa-associated lymphoid tissue (MALT) lymphoma with B-cell origin (Fig. 1E and F). Tissues were formalin-fixed and paraffin-embedded to be used for immunohistochemistry and routine hematoxylin-eosin staining. A histological diagnosis was performed according to the WHO classification (5). In addition, a relapse of the salmon pink-like lesion of the left inferior conjunctiva was also found (Fig. 1G), and MALT lymphoma was diagnosed by histopathological findings (Fig. 1H and I). Continuous imaging sections on the sinus CT scan revealed a soft-tissue mass filled lacrimal duct and sac (Fig. 2A; red arrows). The homology of the MALT lymphoma in the nasal cavity and conjunctiva was confirmed using polymerase chain reaction (PCR) and evidenced by the sharing of the same $\mathrm{IgH}$ gene rearrangement. IgH-rearrangement was not present in the conjunctival lesion in 2004 (Fig. 2B).

A series of systemic surveys revealed several visible lymph nodes in the bilateral submanibular spaces and neck region (Fig. 2C; white arrows). The blood examination revealed elevated IgG for cytomegalovirus and Epstein-Barr virus. In addition, specimens from the conjunctival and nasal MALT lymphoma were negative for Helicobacter pylori and Chlamydia by staining (data not shown). To avoid radiation-related complications and for the conjunctival mass, topical ciloxan ( $0.3 \%$ ciprofloxacin; 4 times a day; Alcon, Fort Worth, TX, USA) and betamethasone eye ointment (for use before sleep; Shionogi \& Co., Ltd., Tokyo, Japan) were provided, and the mass had regressed 1 month later (Fig. 2D). For the nasolacrimal mass and enlarged lymph nodes, the patient received two phases of radiotherapy with a total dosage of 46.8 Gray (Gy) (phase I, 180 cGyx10 covering the left lacrimal sac, nasal cavity and neck; phase II, 180 cGyx16 over the bilateral neck region). Subsequent to radiotherapy, the nasal cavity and lacrimal sac were noted to be tumor free (Fig. 2E; upper; red arrows), and one stationary neck lymph node (Fig. 2E; lower; white arrow) regressed the following year. The patient was regularly followed up at the outpatient Departments of Ophthalmology, Otolaryngology and Oncology of Wan Fang Medical Center, Taipei Medical University. The frequency of visits was every 3 months. No recurrence was found in the subsequent 3 years.

The present study was approved by the Joint Institutional Review Board of Taipei Medical University and written informed consent was obtained from the patient for participation in the study.

\section{Discussion}

To the best of our knowledge, the current study presents the first case report of an IgH-rearranged conjunctival MALT lymphoma with nasal cavity spread through the nasolacrimal duct. It was reported in the literature that $92 \%$ of ophthalmic MALT lymphoma patients have a primary ocular adnexal lymphoma, $60 \%$ of which are primarily localized in the orbit and $33 \%$ of which in the conjunctiva with a salmon-colored appearance $(1,2)$. Conjunctival MALT lymphoma derived from lymphoid hyperplasia is rare due to the benign course of lymphoid hyperplasia $(2,6)$. In the present case, although the conjunctival MALT lymphoma (Fig. 1G-I) appeared following a spontaneous regressed atypical lymphoid hyperplasia (Fig. 1A-C), PCR demonstrated a discordant lymphoproliferative tumor of the left conjunctiva in November 2004 and October 2008 (Fig. 2B).

Genetic rearrangement $(7,8)$ and chronic infectious stimulation $(8,9)$ result in the activation of nuclear factor $\kappa$-light-chain-enhancer of activated B-cells, and subsequently contributes to the malignant transformation of MALT lymphomas (10). Chromosomal abnormalities involving IgH gene translocation, including $\mathrm{t}(1 ; 14)(\mathrm{p} 22 ; \mathrm{q} 32), \mathrm{t}(14 ; 18)$ (q32; 21$)$, and $\mathrm{t}(3 ; 14)(\mathrm{p} 14 ; \mathrm{q} 32)$, are frequently associated with gastric or pulmonary MALT lymphomas but seldom found in conjunctival MALT lymphomas $(1,8)$. In contrast with $\operatorname{IgH}$ rearrangement, infectious agents associated with MALT lymphomas are more common including $H$. pylori, Chlamydia and hepatitis $\mathrm{C}$ virus. Infection with these pathogens and infectious antigen stimulation of conjunctival tissue is not uncommon during life (9). Different from gastric MALT lymphomas, the prevalence of $H$. pylori infection is relatively low in the conjunctiva (11); however, Chlamydia and hepatitis $\mathrm{C}$ virus infections are frequently associated with conjunctival MALT lymphomas $(9,12)$. In the present study, the patient suffered a rare conjunctival MALT lymphoma with IgH rearrangement (Fig. 2B), but tested negative for $H$. pylori and Chlamydia infection. Notably, although $H$. pylori and Chlamydia were undetectable in the conjunctival and nasal MALT lymphomas, the conjunctival tumor lesion showed a good response to topical antibiotics therapy (Fig. 2D), indicating that an atypical infection played a role in the present case.

Ophthalmic MALT lymphoma is a malignancy with a indolent course, and neither the clinical stage, ocular region 
localization, nor therapeutic strategy affects the 5-year survival or progression-free survival rate, with the exception of IgH translocation involvement $(1,2)$. For an IgH-translocated ophthalmic MALT lymphoma, aggressive treatment is necessary due to the high-risk of tumor progression (13). MALT lymphomas are sensitive to external beam radiation, chemotherapy, cryotherapy and oral doxycycline for Chlamydia infection $(1,9)$, and the systemic anti-CD20 antibody, rituximab, is reserved for refractory MALT lymphomas (14). In the present case, to avoid radiation-related ocular complications, a topical ciprofloxacin and steroid were chosen to treat the conjunctival MALT lymphoma, and radiotherapy was given covering bilateral neck, left nasal cavity, and left lacrimal sac. Ciprofloxacin is a broad-spectrum antibiotic covering atypical infections with a low resistance rate, chosen for the present patient, who was negative for H.pylori and Chlamydia infection. The current study demonstrated that a combination of radiation therapy, topical ciprofloxacin and a topical steroid was an effective therapeutic strategy for an IgH-rearranged conjunctival MALT lymphoma with nasal cavity dissemination (Fig. 2D and E).

In summary, a single-clone MALT lymphoma in the conjunctiva with nasal cavity dissemination is rare, but possible. In patients diagnosed with conjunctival MALT lymphoma, thorough clinical examination should be performed to exclude the possibility of conjunctival tumor spreading via the nasolacrimal duct. Otolaryngologist may play a role in nasal examination. The nasolacrimal duct may serve as a channel for conjunctival tumor spreading. IgH-involved translocation is a disease progression factor, and combination therapeutic approach, including topical antibiotics for conjunctival tumor and radiotherapy for extra conjunctival lesions, may successfully control high-risk, disseminated MALT lymphomas.

\section{Acknowledgements}

The authors acknowledge Dr Chia-Che Wu (Ear, Nose, Throat consultant), Dr Jo-Ting Tsai (Oncologist), Dr Gia-Yaun Chen (Radiologist) and Dr Phui-Ly Liew (Pathologist) as clinical consultants at Wan Fang Medical Center, Taipei Medical University.

\section{References}

1. Sjö LD: Ophthalmic lymphoma: Epidemiology and pathogenesis. Acta Ophthalmol 87 Thesis 1: 1-20, 2009.

2. Ferry JA, Fung CY, Zukerberg L, Lucarelli MJ, Hasserjian RP, Preffer FI and Harris NL: Lymphoma of the ocular adnexa: A study of 353 cases. Am J Surg Pathol 31: 170-184, 2007.

3. Cuadra-Garcia I, Proulx GM, Wu CL, Wang CC, Pilch BZ, Harris NL and Ferry JA: Sinonasal lymphoma: A clinicopathologic analysis of 58 cases from the massachusetts general hospital. Am J Surg Pathol 23: 1356-1369, 1999.

4. Yang QP, Zhang WY, Yu JB, Zhao S, Xu H, Wang WY, Bi CF, Zuo Z, Wang XQ, Huang J, et al: Subtype distribution of lymphomas in southwest China: Analysis of 6,382 cases using WHO classification in a single institution. Diagn Pathol 6: 77, 2011.

5. Swerdlow SH, Campo E, Harris NL, Jaffe ES, Pileri SA, Stein H, Thiele J and Vardiman JW (eds): WHO Classification of Tumours of Haematopoietic and Lymphoid Tissues. 4th edition. IARC Press, Lyon, 2008

6. Demirci H, Shields CL, Karatza EC and Shields JA: Orbital lymphoproliferative tumors: Analysis of clinical features and systemic involvement in 160 cases. Ophthalmology 115: $1626-1631,2008$

7. Kossakowska AE, Eyton-Jones S and Urbanski SJ: Immunoglobulin and T-cell receptor gene rearrangements in lesions of mucosa-associated lymphoid tissue. Diagn Mol Pathol 2: 233-240, 1993.

8. Ferreri AJ, Dolcetti R, Du MQ, Doglioni C, Resti AG, Politi LS, De Conciliis C, Radford J, Bertoni F, Zucca E, et al: Ocular adnexal MALT lymphoma: An intriguing model for antigen-driven lymphomagenesis and microbial-targeted therapy. Ann Oncol 19: 835-846, 2008.

9. Verma V, Shen D, Sieving PC and Chan CC: The role of infectious agents in the etiology of ocular adnexal neoplasia. Surv Ophthalmol 53: 312-331, 2008.

10. Isaacson PG and Du MQ: MALT lymphoma: From morphology to molecules. Nat Rev Cancer 4: 644-653, 2004.

11. Ferreri AJ, Ponzoni M, Viale E, Guidoboni M, Conciliis CD, Resti AG, Politi L, Lettini AA, Sacchetti F, Dognini G, et al: Association between Helicobacter pylori infection and MALTtype lymphoma of the ocular adnexa: Clinical and therapeutic implications. Hematol Oncol 24: 33-37, 2006.

12. Ferreri AJ, Guidoboni M, Ponzoni M, De Conciliis C, Dell'Oro S, Fleischhauer K, Caggiari L, Lettini AA, Dal Cin E, Ieri R, et al: Evidence for an association between Chlamydia psittaci and ocular adnexal lymphomas. J Natl Cancer Inst 96: 586-594, 2004.

13. Matsuo $\mathrm{T}$ and Yoshino T: Long-term follow-up results of observation or radiation for conjunctival malignant lymphoma. Ophthalmology 111: 1233-1237, 2004.

14. Salepci T, Seker M, Kurnaz E, Guler DO, Bilici A, Dane F, Aliustaoglu M, Atesoglu EB, Gumus M and Yaylaci M: Conjunctival malt lymphoma successfully treated with single agent rituximab therapy. Leuk Res 33: e10-e13, 2009. 